Lasers in Surgery and Medicine

\title{
Selective Photothermolysis to Target Sebaceous Glands: Theoretical Estimation of Parameters and Preliminary Results Using a Free Electron Laser
}

\begin{tabular}{|c|c|}
\hline Journal: & Lasers in Surgery \& Medicine \\
\hline Manuscript ID: & LSM-08-0237.R1 \\
\hline Wiley - Manuscript type: & Research Article \\
\hline Date Submitted by the Author: & $\mathrm{n} / \mathrm{a}$ \\
\hline Complete List of Authors: & $\begin{array}{l}\text { Sakamoto, Fernanda; Wellman Center for Photomedicine - Massachusetts } \\
\text { General Hospital - Harvard Medical School, Dermatology } \\
\text { Doukas, Apostolos; Wellman Center for Photomedicine - Massachusetts } \\
\text { General Hospital - Harvard Medical School, Dermatology } \\
\text { Farinelli, William; Wellman Center for Photomedicine - Massachusetts } \\
\text { General Hospital - Harvard Medical School, Dermatology } \\
\text { Tannous, Zeina; Wellman Center for Photomedicine - Massachusetts } \\
\text { General Hospital - Harvard Medical School, Dermatology } \\
\text { Shinn, Michelle; Thomas Jefferson National Accelarator Facility } \\
\text { Benson, Steve; Thomas Jefferson National Accelarator Facility } \\
\text { Williams, Gwyn; Thomas Jefferson National Accelarator Facility } \\
\text { Gubeli III, Joseph; Thomas Jefferson National Accelarator Facility } \\
\text { Dylla, H. Frederick; American Institute of Physics } \\
\text { Anderson, R. Rox; Wellman Center for Photomedicine, Massachusetts } \\
\text { General Hospital }\end{array}$ \\
\hline Key Words: & $\begin{array}{l}\text { acne, free electron laser, in vitro, light, laser, therapy, spectroscopy, near } \\
\text { infrared, Monte Carlo Method }\end{array}$ \\
\hline
\end{tabular}

\section{SCHOLARONE \\ Manuscripts}


Selective Photothermolysis to Target Sebaceous Glands: Theoretical Estimation of Parameters and Preliminary Results Using a Free Electron Laser.

Fernanda H. Sakamoto, M.D., Ph.D. (1,2), Apostolos G. Doukas, Ph.D. (1,2), William A. Farinelli, B.A. (1,2), Zeina Tannous, M.D. (1,2), Michelle Shinn, Ph.D. (3), Steve Benson, Ph.D. (3), Gwyn P. Williams, Ph.D. (3), Joseph F. Gubeli III, M.S. (3), H. Frederick Dylla, Ph.D. (4), R. Rox Anderson, M.D. (1,2).

1. Wellman Center for Photomedicine, Department of Dermatology, Massachusetts General Hospital, Boston, MA, USA, 02114.

2. Harvard Medical School, Department of Dermatology, Boston, MA, USA, 02114

3. Thomas Jefferson National Accelerator Facility, Newport News, VA, USA, 23606.

4. American Institute of Physics, College Park, MD 20740.

Corresponding author: R. Rox Anderson, MD.

55 Fruit St. BHX 630

02114 Boston, MA, USA

Phone: +1-617-7266168 Fax: +1-617-7266121

E-mail: rranderson@partners.org

Word count: 4,286 (core text)

Key Words: Acne, free electron laser, in vitro, light, laser, therapy, spectroscopy, near infrared, Monte Carlo Method.

Research Support: Medical Free Electron Laser Research Program. AFOSR, US

Department of Defense, Contract grant number: FA 9550-04-1-0079; Contract grant sponsor: Office of Naval Research: Contact grant sponsor: Commonwealth of Virginia. 


\begin{abstract}
.
Background and Objectives: The success of permanent laser hair removal suggests that selective photothermolysis (SP) of sebaceous glands, another part of hair follicles, may also have merit. About $30 \%$ of sebum consists of fats with copious $\mathrm{CH}_{2}$ bond content. SP was studied in vitro, using free electron laser (FEL) pulses at an infrared $\mathrm{CH}_{2}$ vibrational absorption wavelength band.
\end{abstract}

Methods: Absorption spectra of natural and artificially prepared sebum were measured from $200 \mathrm{~nm}$ to $3000 \mathrm{~nm}$, to determine wavelengths potentially able to target sebaceous glands. The Jefferson National Accelerator superconducting FEL was used to measure photothermal excitation of aqueous gels, artificial sebum, pig skin, human scalp and forehead skin (sebaceous sites). In vitro skin samples were exposed to FEL pulses from 1620 to $1720 \mathrm{~nm}$, spot diameter 7-9.5 mm with exposure through a cold $4^{\circ} \mathrm{C}$ sapphire window in contact with the skin. Exposed and control tissue samples were stained using H\&E, and nitroblue tetrazolium chloride staining (NBTC) was used to detect thermal denaturation.

Results: Natural and artificial sebum both had absorption peaks near 1210, 1728, 1760, 2306 and $2346 \mathrm{~nm}$. Laser-induced heating of artificial sebum was approximately twice that of water at 1710 and $1720 \mathrm{~nm}$, and about $1.5 \mathrm{x}$ higher in human sebaceous glands than in water. Thermal camera imaging showed transient focal heating near sebaceous hair follicles. Histologically, skin samples exposed to $\sim 1700 \mathrm{~nm}, \sim 100-125 \mathrm{~ms}$ pulses showed evidence of selective thermal damage to sebaceous glands. Sebaceous glands were positive for NBTC staining, without evidence of selective loss in samples exposed to the laser. Epidermis was undamaged in all samples. 
1

2

3

4

5

6

7

8

9

10

11

12

13

14

15

16

17

18

19

20

21

22

23

24

25

26

27

28

29

30

31

32

33

34

35

36

37

38

39

40

41

42

43

44

45

46

47

48

49

50

51

52

53

54

55

56

57

58

59

60
Conclusions: SP of sebaceous glands appears to be feasible. Potentially, optical pulses at $\sim 1720 \mathrm{~nm}$ or $\sim 1210 \mathrm{~nm}$ delivered with large beam diameter and appropriate skin cooling in approximately $0.1 \mathrm{~s}$ may provide an alternative treatment for acne. 


\section{Introduction}

Selective Photothermolysis (SP) has been used to treat many different disorders including glaucoma, gastrointestinal lesions, vascular malformations, vocal cord lesions, verruca, pigmented lesions, tattoos, and hair follicles (1). In general, specific chromophores of the tissue are necessary (such as hemoglobin, melanin, etc) for SP to selectively deposit energy in or near the target. Appropriate source wavelength, beam optics, and pulse duration nearly equal to the thermal relaxation time of the desired target structure are generally required $(2,3)$. Static or dynamic tissue cooling can be applied to protect adjacent tissues from thermal injury (4).

Acne is the most common skin disease and the most common cause of permanent facial scarring (5-7). For the treatment of acne, lasers have been used with wavelengths of $1450 \mathrm{~nm}(8)$ (9), 585-595 nm (PDL, pulsed dye lasers) (10-13), and $535 \mathrm{~nm}$ (KTP) (14). None of these wavelengths are preferentially absorbed by sebaceous glands, but may improve acne to some extent. Statistically significant reduction of lesion count and severity with histological thermal damage in the upper dermis was observed using 1450 $\mathrm{nm}$ (8). This wavelength is well absorbed by water, inducing an improvement effect in acne by unknown mechanisms. Mechanism of action and efficacy using PDL and KTP lasers are controversial (11-13), and may involve SP of microvessels. No studies of laser or light treatments have shown convincing evidence to improve non-inflammatory acne. To date, laser treatment of acne is limited, expensive and requires multiple sessions.

All medical applications of SP at present are based on electronic absorption transitions; however there is no intrinsic limitation to using vibrational or other excitation modes. Anderson et al (15) first described the use of $\mathrm{CH}_{2}$ bond vibrational absorption modes as 
a means for SP of lipid-rich tissues, identifying two absorption bands near 1210 and $1720 \mathrm{~nm}$. At both wavebands, lipids have somewhat stronger optical absorption than water, but the contrast is weak. Of these wavelengths, $1210 \mathrm{~nm}$ has deeper tissue penetration. It was shown that $1210 \mathrm{~nm}$ exposures are capable of preferentially damaging subcutaneous fat when delivered in combination with skin cooling. Subcutaneous fat is a thick layer that retains heat well; such that even very long exposures (20 seconds) allow thermal confinement in subcutaneous fat. In contrast, sebaceous glands are typically small intradermal structures (diameters 100-500 $\mu \mathrm{m}$, average $250 \mu \mathrm{m})(16)$, with an estimated thermal relaxation time of about $100-250$ milliseconds or less. While the $1210 \mathrm{~nm}$ wavelength band is well suited for targeting subcutaneous fat due to deeper penetration, in theory, both the $1210 \mathrm{~nm}$ and $1720 \mathrm{~nm}$ wavelength bands could be used for targeting sebaceous glands.

Sebum produced by sebaceous glands could possibly be used as a chromophore for SP. About $30 \%$ of sebum is composed of free fats, triglycerides, squalene, cholesterol and wax esters (17) with copious $\mathrm{CH}_{2}$ bond content. In human tissue, sebaceous glands are located about $1 \mathrm{~mm}$ deep (18). In this pilot study, we studied optical absorption spectra of sebum, measured the wavelength-dependant photothermal excitation of sebum, water and tissue samples, and looked for histological evidence of sebaceous gland SP with optical pulses near $1700 \mathrm{~nm}$.

\section{Materials and Methods}

Spectrophotometry 
A Cary 5000 integrating sphere spectrophotometer (Varian Inc., Walnut Creek, CA, USA) was used for measurements of absorption spectra of natural and artificial sebum and for transmittance/reflectance measurements of human skin from 400 to $2500 \mathrm{~nm}$.

\section{Human skin spectroscopy}

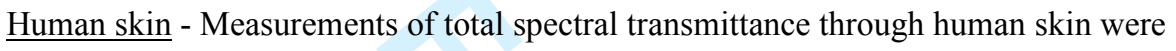
performed using a slightly modified integrating sphere spectrophotometer, conducted on discarded tissue collected from abdominoplasty and face-lifting surgery of three Fitzpatrick skin phototype I-III patients performed at Massachusetts General Hospital after Institutional Review Board approval. Fresh frozen tissue samples 3 x $2 \mathrm{~cm}$ were thawed and subcutaneous fat was removed leaving approximately $2 \mathrm{~mm}$-thick samples, containing full thickness of epidermis and dermis. Tissue was placed on a microscope cover slip (0.17 mm thick, 35 x $60 \mathrm{~mm}$, Gold Seal ${ }^{\circledR}$ Cover Glass, C2008 Thermo Fisher Scientific Inc. Portsmouth, NH, USA) with epidermis facing the spectrophotometer light beam, at the entrance port of the integrating sphere. Skin transmittance and reflectance were measured every $2 \mathrm{~nm}$ from 400-2500 $\mathrm{nm}$ in a single-beam measurement mode, using the microscope cover slip and lens assembly alone as reference.

\footnotetext{
Absorption spectra of sebum

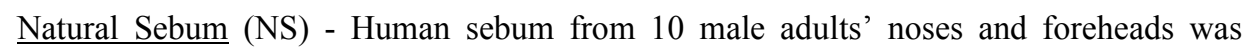
collected on microscope slides 1 hour after the skin was cleansed with water, soap and thorough rinsing. Ages varied from 25 to 64 years old (median 35 years old). The material collected was dissolved in carbon tetrachloride $\left(\mathrm{CCl}_{4}\right)$ (Sigma-Aldrich, Saint
} 
Louis, MO, USA), and studied by absorption spectrophotometry set to measure every 2 $\mathrm{nm}$ from 200-3000 nm, using $\mathrm{CCl}_{4}$ as reference. This yielded qualitative absorption spectra in the near and mid-infrared region for human sebum.

Artificial Sebum (AS) - A mix of artificial sebum lipids was prepared according to Nordstrom et al.'s report of sebum content (17) using 4\% cholesterol (Sigma-Aldrich, Saint Louis, MO, USA), 25\% wax ester (palmitic acid stearyl ester, Sigma-Aldrich, Saint Louis, MO, USA), 20\% squalene (Sigma-Aldrich, Saint Louis, MO, USA), 16\% triglycerides (tripalmitin, Sigma-Aldrich, Saint Louis, MO, USA) and 33\% free-fatty acid (palmitic acid free-acid, Sigma-Aldrich, Saint Louis, MO, USA). The artificial sebum lipid was a translucent material that was dissolved in $\mathrm{CCl}_{4}$ to obtain a transparent solution for absorption spectrophotometry. A total of $0.4 \mathrm{~g}$ of AS was diluted into $1 \mathrm{~mL}$ of $\mathrm{CCl}_{4}$, measured in a $1 \mathrm{~mm}$ cuvette against $\mathrm{CCl}_{4}$ as the reference.

\section{Monte Carlo modeling}

A Monte Carlo model of light interaction with tissue was used (19), to analyze the spectrophotometry measurements of ex vivo skin. The input data was total transmittance (Tt), collimated transmittance (Tc), diffuse reflectance (Rd) and skin sample thickness. The Henyey-Greestein scattering phase function with an average cosine, $g$, was set to 0.9 (20). Refractive index, $n$, of tissue was set to be 1.4 (21). Simulation was performed at $1210 \mathrm{~nm}$ and 1720 at $1.0 \mathrm{~mm}$ and $2.0 \mathrm{~mm}$ deep, corresponding to estimated $\mathrm{SG}$ and fat depth of location, using the inverse adding-doubling (IAD) method (22). Absorption coefficient $\mu_{a}$ and scattering coefficient $\mu_{s^{\prime}}$ of skin were, then, calculated. 


\section{Free Electron Laser (FEL)}

The superconducting electron accelerator-driven FEL from the Thomas Jefferson National Accelerator Laboratory was adjusted to allow wavelength tuning and broad output power range up to $1000 \mathrm{~W}$. The FEL produces a train of 500-1700 femtosecond pulses (FWHM), which in accordance with Heisenberg's principle produces a broad wavelength bandwidth that typically varied from $15-25 \mathrm{~nm}(23,24)$. Spectrometer scans were obtained at each wavelength. The set up and bandwidth were similar to that reported by Anderson et al. (15), but dielectric mirrors were used in the laser cavity in 
order to tune laser wavelengths from 1620 to $1720 \mathrm{~nm}$, and metal mirrors were used from 1600 to $1800 \mathrm{~nm}$. FEL beam diameter at the sample was adjusted with lenses to a range of 7.0-9.5 mm round spots. An aperture was used to improve beam uniformity by removing the edges of the Gaussian beam profile. The estimated incident exposure beam uniformity on samples was $\pm 30 \%$. FEL exposure duration was controlled at the accelerator level, by adjusting the electron source duration from 25-250 ms.

\section{Photothermal excitation measurements}

FEL-induced temperature rise was measured at the surface of human tissue samples and other samples (artificial sebum lipids, aqueous gels) by photothermal radiometry using a 3 5 $\mu \mathrm{m}$ thermal camera (PM180 Thermacan, FLIR Systems, Inc., N. Billerica, MA, USA) calibrated for the experiment, during FEL exposure of the samples. Camera software includes peak-temperature detection at a given location in the field of view, which was aligned with the center of the FEL beam incident upon a sample's surface. The thermal camera was blind to the FEL wavelengths. The maximum temperature was limited to less than $65^{\circ} \mathrm{C}$ to minimize tissue thermal damage for this part of the experiment. Photothermal excitation was normalized for beam energy by dividing each exposure's induced peak temperature rise of the sample $(\Delta \mathrm{T})$, by the associated FEL exposure fluence (E) (result expressed in ${ }^{\circ} \mathrm{C} \cdot \mathrm{J}^{-1} \cdot \mathrm{cm}^{2}$ ). The mean and standard deviation (SD) of 6 independent measurements of the photothermal excitation were determined for each sample at each wavelength.

Statistical analysis of photoexcitation measurements was performed using SPSS Version 15. (SPSS Inc., Chicago, IL, USA), and Microcal ${ }^{\mathrm{TM}}$ Origin $^{\circledR}$ Version 6.0 (Microcal Software Inc., Northampton, MA, USA). 


\section{Study design}

Initially, the photothermal excitations for human facial skin, pig skin, artificial sebum and water were determined for wavelengths from $1620 \mathrm{~nm}$ to $1720 \mathrm{~nm}$ (increasing $20 \mathrm{~nm}$ per exposure) at $25 \mathrm{~ms}$ pulse duration. Six measurements for each wavelength were taken for statistical validation. An optimal wavelength region was defined as the wavelength which produced statistically significant greater thermal rise for the sebaceous glands/sebum samples over water gels/dermis samples.

In the second part of the experiment, human and porcine skin samples were exposed to FEL pulses ranging systematically from 100-185 milliseconds (ms) at the $\sim 1700 \mathrm{~nm}$ wavelength determined from photothermal excitation measurements to be promising for SP of sebaceous glands. Human skin samples were either prepared with their top removed to expose the glands as described, or intact. For intact skin samples, the dermal side was placed on a warm $\left(37^{\circ} \mathrm{C}\right)$ plate, and the FEL was delivered through a cold sapphire window pressed onto the epidermal surface, as previously described (15) Briefly, the sapphire window ( $3 \mathrm{~mm}$ thickness $\mathrm{x} 50 \mathrm{~mm}$ diameter) was mounted in thermal contact with a copper block through which ice water was passed by means of a small pump, maintaining the window temperature of $6-8{ }^{\circ} \mathrm{C}$.

The cold contact sapphire window was used to protect overlying epidermis and superficial dermis during exposures. Samples were temperature equilibrated for at least 30 seconds prior to each FEL exposure. At least 6 samples were exposed to each of a range of FEL fluences at each wavelength and pulse duration, to allow histological examination of independent, repeated samples. 
The measured fluence delivered to samples ranged from 64 to $120 \mathrm{~J} / \mathrm{cm}^{2}$. The precision of pulse-to-pulse fluence measurement was approximately $\pm 5 \%$. However, the absolute calibration error of the absolute FEL incident fluence was estimated to be up to $\pm 50 \%$ due to the combined uncertainties of energy measurement, beam diameter and beam profile variations in the exposure area.

\section{Tissue Thermal Effects Assessment}

Exposed and unexposed control tissue samples were bisected with a razor blade through the optical exposure axis. One half was processed for routine light microscopy using hematoxylin and eosin staining (H\&E) and for polarized light microscopy. Loss of birefringence is a marker of type I collagen thermal damage when denaturation occurs at $70{ }^{\circ} \mathrm{C}$. (25). The other half was incubated for 15 minutes at room temperature in a solution containing nitroblue tetrazolium chloride (NBTC) according to Neumann's technique for visualizing activity of NADPH diaphorase, a thermally sensitive enzyme (26). Cell lethality caused by thermal denaturation is well correlated with loss of enzyme activity, and with consequent loss of NBTC dark blue staining.

Histological evidence of tissue changes was blindly assessed by a dermatopathologist, who compared FEL exposed with unexposed control sites.

\section{Results}

\section{Absorption spectra}

Quantitative absorption spectra are shown in figure 1, for artificial sebum and water. Natural sebum showed weak absorption maxima at $1212 \mathrm{~nm}$ and $1364 \mathrm{~nm}$, stronger maxima at $\sim 1720$ and $1760 \mathrm{~nm}$, and strong absorption at 2306 and $2346 \mathrm{~nm}$. Due to the 
very small amount of sebum collected, a quantitative spectrum was not possible for human sebum. Absorption spectra of artificial sebum was qualitatively very similar to natural sebum, with absorption maxima at $1210 \mathrm{~nm} ; 1390$ and $1414 \mathrm{~nm} ; 1728$ and 1760; 2306 and $2346 \mathrm{~nm}$. At $1210 \mathrm{~nm}$, artificial sebum lipid absorption was measured to be nearly equal to that of water (figure 1); while artificial sebum lipids had higher absorption than water at $1726 \mathrm{~nm}, 2306 \mathrm{~nm}$ and at $2346 \mathrm{~nm}$.

When optical absorption by the $70 \%$ water content and $30 \%$ lipids content of sebum in vivo is considered (17), the resultant spectrum shown in figure 1 is obtained. At $1726 \mathrm{~nm}$, in vivo sebum is estimated to have 1.20 times the absorption of water.

\section{Size of human sebaceous glands}

The length and width of human facial skin sebaceous glands were $0.26 \mathrm{~mm}(\mathrm{SD} \pm 0.11)$, and $0.12(\mathrm{SD} \pm 0.12) \mathrm{mm}$ respectively. Facial sebaceous glands were found from 0.12 to $0.76 \mathrm{~mm}$ deep from the surface of the skin; average of $0.50 \mathrm{~mm}(\mathrm{SD} \pm 0.19)$.

\section{Monte Carlo modeling}

At $1200 \mathrm{~nm}$, calculated $\mu_{a}=1.22( \pm 0.21) \mathrm{cm}^{-1}$ and $\mu_{s^{\prime}}=14.45( \pm 2.24) \mathrm{cm}^{-1}$. At $1720 \mathrm{~nm}$, calculated $\mu_{a}=9.63( \pm 2.02) \mathrm{cm}^{-1}$ and $\mu_{s^{\prime}}=9.78( \pm 1.46) \mathrm{cm}^{-1}$. Table 1 summarizes results of Monte Carlo simulation.

\section{Estimation of exposure parameters to target sebaceous glands}

The local temperature rise $(\Delta \mathrm{T})$ within tissue from absorption of a short optical pulse, ignoring heat diffusion during the pulse, can be estimated as $\Delta \mathrm{T}=\mu_{\mathrm{a}} \mathrm{F}_{\mathrm{o}} T /(\rho \mathrm{c})$ where $\mu_{\mathrm{a}}$ is 
the local optical absorption coefficient (typically in units of $\mathrm{cm}^{-1}$ ), $\mathrm{F}_{\mathrm{o}}$ is the incident optical fluence at the tissue surface (typically in units of $\mathrm{J} / \mathrm{cm}^{2}$ ), $T$ is the effective optical transmittance through tissue to the site of interest, $\rho$ is density and $\mathrm{c}$ is specific heat capacity. The density and heat capacity of water-rich tissues such as epidermis and dermis are very close to those of water, while density and heat capacity of lipid-rich tissues such as fat and sebaceous glands are lower. For dermis, $\mathrm{c} \approx 4.2 \mathrm{~J}^{-1} \mathrm{~K}^{-1}$ and $\rho \approx 1$ $\mathrm{g} / \mathrm{cm}^{3}$. For fat, $\rho \mathrm{c} \approx 2.1 \mathrm{JK}^{-1} \mathrm{~cm}^{-3}$, half that of dermis (27). For sebaceous glands with $30 \%$ lipid content (17), $\rho \mathrm{c}$ is estimated to be approximately $3.6 \mathrm{JK}^{-1} \mathrm{~cm}^{-3}$.

The optimal pulse duration for SP is typically equal to or less than the thermal relaxation time $(\tau)$ of the target structure, in this case sebaceous glands (2). In units of seconds and millimeters for biological targets, $\tau \approx$ diameter $^{2}$. According to our measurements of human facial sebaceous glands in this study, their thermal relaxation time ranges from about 0.020 to 0.129 seconds, with a mean of about $0.06 \mathrm{~s}$. In theory, selective photothermolysis of sebaceous glands would be expected from appropriate optical pulses of about 0.1 second or $100 \mathrm{~ms}$.

To estimate the incident fluence $\left(\mathrm{F}_{\mathrm{o}}\right)$ expected for SP, the temperature rise $(\Delta \mathrm{T})$ should be estimated. The baseline temperature $\left(\mathrm{T}_{\mathrm{SG}}\right)$ at the $\mathrm{SG}$ level $(\sim 0.5-1.0 \mathrm{~mm}$ depth) and subcutaneous fat $(\sim 2.0 \mathrm{~mm}$ depth$)$ while a cold window is applied for epidermal protection at $4^{\circ} \mathrm{C}$ was estimated, we considered Fourier's law integrated for a simple exponential situation, where uniform temperature across equally sized end surfaces and perfectly insulated sides exist, and the heat flow rate between the end surfaces is given by: 
Where $\Delta Q / \Delta t$ is the heat flow by a given time period, $k$ is the material conductivity, $A$ is the cross-sectional surface area, $\Delta \mathrm{T}$ is the temperature difference between the ends, and $\Delta x$ is the distance between the ends.

When cooling is applied in a large enough area until an equilibrium state, it behaves as a two dimension model, where: $\mathrm{T}_{\text {surf }}$, is the temperature at the surface (cooling plate at $\left.4^{\circ} \mathrm{C}\right), \mathrm{T}_{b}$ is the baseline body temperature at the subcutaneous fat level $\left(37^{\circ} \mathrm{C}\right), \mathrm{T}_{\mathrm{SG}}$ is the baseline temperature at the SG level, $x_{\mathrm{b}}$ is the distance from the surface to the top of subcutaneous fat $(2 \mathrm{~mm}), x_{\mathrm{SG}}$ is the distance from the surface to the SG. From the simple exponential Fouries's law:

$$
\mathrm{T}_{\mathrm{SG}}=\underline{x}_{x_{\mathrm{bG}}}\left(\mathrm{T}_{\mathrm{b}}-\mathrm{T}_{\mathrm{surf}}\right)+\mathrm{T}_{\text {surf }}
$$

Using the simplified heat equation, the estimated baseline temperature at the sebaceous glands inside the tissue samples of this study was approximately $12^{\circ} \mathrm{C}$.

For most mammalian cells such as SG cells heated for 0.1-1 second, cell lethality is achieved at temperatures of about $55-65^{\circ} \mathrm{C}$. The temperature rise $\left(\Delta \mathrm{T}_{\mathrm{SG}}\right)$ expected for thermal damage to $\mathrm{SG}$ in this study is therefore about $50^{\circ} \mathrm{C}$.

Parameters for the demonstrated and theoretical selective photothermolysis of lipid rich tissues are summarized in table 1.

\section{FEL Photothermal Excitation}

Photothermal excitation measurements are summarized in figure 2. At fixed pulse duration (25 ms), the three different samples (human sebaceous glands, AS and water) 
showed statistically different photothermal excitation $(\mathrm{p}<.05$, Tukey and Scheffe tests). Laser induced transient heating of the artificial sebum was approximately $2.3 \mathrm{x}$ that of the aqueous gel at $1700 \mathrm{~nm}$, and about 1.4x higher in human sebaceous glands in situ than in the aqueous gel. At 1720, nm heating of artificial sebum was 2.1x higher than aqueous gel and 1.6x higher in human sebaceous glands in situ than the gel.

As a preferential photothermal excitation of artificial sebum and human sebaceous glands was produced at about $1700-1720 \mathrm{~nm}$, we measured sebaceous glands photothermal excitation with different pulse widths at an FEL wavelength of $\sim 1700 \mathrm{~nm}$.

Thermal camera images of the samples sectioned horizontally $\sim 1 \mathrm{~mm}$ deep showed preferential thermal excitation in small dots corresponding to follicles when exposed to FEL at 1700-1720 $\mathrm{nm}$. When wavelength was fixed at $1700 \mathrm{~nm}$, "hot-spots" were apparent and obvious near hair follicles at the surface of tissue for pulse width up to 100 - $125 \mathrm{~ms}$. At $1700 \mathrm{~nm}$, the contrast between of photothermal excitation of sebaceous glands, with that of adjacent tissue, decreased for exposures $>100 \mathrm{~ms}$.

\section{Microscopy: NBTC Staining}

NBTC staining in unexposed control samples was strong and variable in sebaceous follicles, which made it difficult to assess changes in SG staining in the exposed samples. There was non-selective or complete loss of dermal NBTC staining after FEL exposure $(1700 \mathrm{~nm})$ at pulse widths higher than and equal to $135 \mathrm{~ms}$, corresponding to FEL fluence 87.6 to $120 \mathrm{~J} / \mathrm{cm}^{2}$. At 100 and $125 \mathrm{~ms}$, weak NBTC staining could be observed in the epidermis and dermis at the laser exposed sites, but it was difficult to determine if there was decreased staining in sebaceous glands (figure 3). 


\section{Microscopy: H\&E Staining}

Blind assessment of H\&E-stained histopathology sections of FEL-exposed full thickness human sebaceous skin showed no changes in the epidermis and upper dermis for FEL exposures up to $\sim 120 \mathrm{~J} / \mathrm{cm}^{2}$ delivered in $175 \mathrm{~ms}$, at and above which there was subepidermal blister formation consistent with thermal injury (Figure 4A). At FEL exposures of 100 and $125 \mathrm{~ms}\left(64-70 \mathrm{~J} / \mathrm{cm}^{2}\right.$ at $100 \mathrm{~ms}$, and $78-82 \mathrm{~J} / \mathrm{cm}^{2}$ at $\left.125 \mathrm{~ms}\right)$ no epidermal or superficial dermal injury was seen. At these exposures, histological changes were limited to sebaceous glands and sebaceous follicles. There was thickening and altered staining of collagen bundles around the sebaceous follicles, loss of sebocytes structural arrangement and nuclear changes consistent with thermal injury to pilosebaceous units (PSU) (Figure 5B and 5C). Samples that were exposed to pulses longer than and equal to $135 \mathrm{~ms}\left(87.6-93.5 \mathrm{~J} / \mathrm{cm}^{2}\right)$ showed extensive non-selective thermal denaturation of dermis (Figure 5D).

Loss of birefringence is a marker of thermal damage to dermal collagen. Reduction of collagen birefringence was observed after $1700 \mathrm{~nm}$ FEL exposures longer than or equal to $135 \mathrm{~ms}$ (Figure 5B).

\section{Discussion}

The primary new findings of this study are: (1) the determination of optical absorption spectra for sebum in the near infrared; (2) determination of optimal wavelengths for selective photothermolysis to target sebaceous glands, at approximately $1210 \mathrm{~nm}, 1726$ $\mathrm{nm}$ and $1760 \mathrm{~nm}$ [the strong 2306 and $2346 \mathrm{~nm}$ absorption band wavelengths do not 
penetrate to the depth SG in vivo] ; (3) estimation of human facial sebaceous glands thermal relaxation time to be about $100 \mathrm{~ms}$; (4) estimation of the parameters for targeting SG using about $50 \mathrm{~J} / \mathrm{cm}^{2}$ incident fluence at $1720 \mathrm{~nm}$; and (5) preliminary evidence by $\mathrm{H} \& \mathrm{E}$ stained histology of selective photothermal targeting of sebaceous glands using an FEL at $1700-1720 \mathrm{~nm}$, with optical pulse widths from 100 to $125 \mathrm{~ms}$ and fluences of $65-82 \mathrm{~J} / \mathrm{cm}^{2}$ delivered with skin cooling to protect the epidermis. These experimental findings are consistent with theoretical values (table 1). However, theory also predicts a relatively narrow range of parameters to achieve SP of sebaceous glands, compared with other known examples of SP.

Anderson et al. (2006) previously reported absorption spectra for subcutaneous fat, and demonstrated photothermal damage of subcutaneous fat using the Jefferson Laboratory FEL near $1200 \mathrm{~nm}$, but the possibility of targeting sebaceous glands was not investigated. Sebum lipids are markedly different from those of fat, and the absorption spectra of sebum in the infrared reported here, are novel. We also demonstrated that natural and artificial sebum have similar absorption spectra. Near infrared absorption by lipids is due to vibrational overtone modes, which are combination frequencies of the fundamental modes found in the mid infrared region (28). Interestingly, both natural and artificial sebum have interesting double peaks that are related to different harmonic vibrations of the same chemical bonds. The absorption bands at wavelengths over $2200 \mathrm{~nm}$ are in the region of C-H combination bands that could correspond to $\mathrm{CH}+\mathrm{CH}, \mathrm{CH}-\mathrm{CC}, \mathrm{CH}_{2}$ or $\mathrm{CH}_{3}$ (28). At about $1700 \mathrm{~nm}$ is the 1 st overtone $\mathrm{C}-\mathrm{H}$ stretching region (with two peaks representing, for example, $\mathrm{CH}$ and $\left.\mathrm{CH}_{2}\right)(28)$. The peaks at about $1400 \mathrm{~nm}$ represent $1^{\text {st }}$ $\mathrm{CH}$ combination overtone modes that could be, for example, $\mathrm{CH}_{2}$ and maybe $\mathrm{CH}_{3}(28)$ ). 
At about $1200 \mathrm{~nm}$, the $2^{\text {nd }}$ overtone $\mathrm{C}-\mathrm{H}$ stretching region, could represent $\mathrm{CH}$ vibration (28). Like fat, both artificial sebum and natural sebum presented peaks near $1210 \mathrm{~nm}$ and at about $1726 \mathrm{~nm}$ and $1760 \mathrm{~nm}$. At these regions, the absorption coefficient of sebum is higher than water (Figure 1).

We used the absorption spectrophotometric data with established models of SP to estimate and to compare with thermal excitation as defined by the mean temperature rise (measured by the thermal camera) per incident $\mathrm{J} / \mathrm{cm}^{2}$, in a manner similar to that reported by Anderson et al (2006) (15).

In theory, selective photothermolysis of sebaceous glands will require optical pulses of about 0.1 second or less using about $245 \mathrm{~J} / \mathrm{cm}^{2}$ at $1210 \mathrm{~nm}$ and about $67 \mathrm{~J} / \mathrm{cm}^{2}$ at 1720 $\mathrm{nm}$, when delivered with contact cooling at $4^{\circ} \mathrm{C}$, in a large-diameter optical beam.

The theoretical estimated values correlated well with results obtained in this pilot study, but an exact comparison is lacking due to experimental and biological variability. It is apparent from both, that there is a narrow range of exposure parameters for which preferential damage of SG is expected. At around 100-125 ms and $60-80 \mathrm{~J} / \mathrm{cm}^{2}$ incident fluence, both H\&E and NBTC stained histopathology samples showed selective thermal damage around sebaceous glands, with vacuolization and disruption of the integrity of the glands. Longer pulses at higher fluences showed non-selective dermal damage, consistent with SP theory. Loss of dermal collagen birefringence is a relatively insensitive marker of dermal injury; even with FEL exposures causing extensive dermal injury by both NBTC and H\&E staining, collagen birefringence was still positive.

It is to be noted that skin samples used for the FEL photoexcitation were collected from scalp and face lifting, which is a surgical procedure usually performed in aged skin; and 
therefore not the ideal samples to study acne skin. Facial skin in adolescents and young adults usually presents SG twice the size of aged skin, which would probably increase the range of pulse duration for SP of sebaceous glands in young adults.

These encouraging preliminary results demonstrate the feasibility of 1700-1720 nm optical pulses to affect sebaceous glands with apparently minimal injury to other skin structures. In theory and in our experiment, the ideal pulse duration is probably about $100 \mathrm{~ms}$, skin cooling is necessary to protect the overlying epidermis and upper dermis, a fluence of greater than $65 \mathrm{~J} / \mathrm{cm}^{2}$ is needed, and there is a narrow fluence range capable of producing selective damage to SG. It should be noted that a source at $1726 \mathrm{~nm}$ with bandwidth narrower than the FEL used in this study maybe superior.

Alexander et al. (29) recently reported a failed attempt to produce SP of sebaceous glands using a Raman-shifted fiber laser at $1708 \mathrm{~nm}$, in vitro. Unfortunately, the conditions studied were far outside the range of appropriate parameters. A narrow beam, at only 2 mm diameter was used, which severely limits the available fluence at the sebaceous glands and favors unwanted injury to the epidermis. The available laser power was merely $4 \mathrm{~W}$, compared with our study using up to $1000 \mathrm{~W}$ from the FEL. The exposure duration studied by Alexander et al. was ten times too long. The reported laser wavelength of $1708 \mathrm{~nm}$ is not ideal for SG targeting and the laser bandwidth was not reported. Finally, the authors presented a data figure obtained from our previous work $(15,30)$ in which they apparently assume that SG lipid absorption is the same as that of subcutaneous fat. Our work here, presents the first detailed absorption spectrum for sebum lipids, which is substantially different from that of fat. 
It remains unclear whether laser pulses near $1720 \mathrm{~nm}$ or $1200 \mathrm{~nm}$ can be configured for safe and effective treatment of acne. This study at least suggests that it is possible. 


\section{References}

1. Anderson RR. Lasers in dermatology--a critical update. J Dermatol 2000; 27(11):700-705.

2. Anderson RR, Parrish JA. Selective photothermolysis: precise microsurgery by selective absorption of pulsed radiation. Science 1983; 220(4596):524-527.

3. Altshuler GB, Anderson RR, Manstein D, Zenzie HH, Smirnov MZ. Extended theory of selective photothermolysis. Lasers Surg Med 2001; 29(5):416-432.

4. Zenzie HH, Altshuler GB, Smirnov MZ, Anderson RR. Evaluation of cooling methods for laser dermatology. Lasers Surg Med 2000; 26(2):130-144.

5. Cunliffe WJ, Gould DJ. Prevalence of facial acne-vulgaris in late adolescence and in adults. Br Med J 1979; 1(6171):1109-1110.

6. Stern RS. Dermatologists and office-based care of dermatologic disease in the 21st century. J Invest Dermatol Symp Proc 2004; 9(2):126-130.

7. Stern RS. The prevalence of acne on the basis of physcal-examination. J Am Acad Dermatol 1992; 26(6):931-935.

8. Paithankar DY, Ross EV, Saleh BA, Blair MA, Graham BS. Acne treatment with a 1,450 nm wavelength laser and cryogen spray cooling. Lasers Surg Med 2002; 31(2):106-114.

9. Friedman PM, Jih MH, Kimyai-Asadi A, Goldberg LH. Treatment of inflammatory facial acne vulgaris with the 1450-nm diode laser: a pilot study. Dermatol Surg 2004; 30(2 Pt 1):147-151.

10. Glaich AS, Friedman PM, Jih MH, Goldberg LH. Treatment of inflammatory facial acne vulgaris with combination 595-nm pulsed-dye laser with dynamiccooling-device and 1,450-nm diode laser. Lasers Surg Med 2006; 38(3):177-180.

11. Orringer JS, Kang S, Hamilton T, Schumacher W, Cho S, Hammerberg C, Fisher GJ, Karimipour DJ, Johnson TM, Voorhees JJ. Treatment of acne vulgaris with a pulsed dye laser: a randomized controlled trial. JAMA 2004; 291(23):2834-2839.

12. Seaton ED, Charakida A, Mouser PE, Grace I, Clement RM, Chu AC. Pulsed-dye laser treatment for inflammatory acne vulgaris: randomised controlled trial. Lancet 2003; 362(9393):1347-1352.

13. Seaton ED, Mouser PE, Charakida A, Alam S, Seldon PM, Chu AC. Investigation of the mechanism of action of nonablative pulsed-dye laser therapy in photorejuvenation and inflammatory acne vulgaris. Br J Dermatol 2006; 155(4):748-755.

14. Baugh WP, Kucaba WD. Nonablative phototherapy for acne vulgaris using the KTP 532 nm laser. Dermatol Surg 2005; 31(10):1290-1296.

15. Anderson RR, Farinelli W, Laubach H, Manstein D, Yaroslavsky AN, Gubeli J, 3rd, Jordan K, Neil GR, Shinn M, Chandler W, Williams GP, Benson SV, Douglas DR, Dylla HF. Selective photothermolysis of lipid-rich tissues: a free electron laser study. Lasers Surg Med 2006; 38(10):913-919.

16. Manuskiatti W, Dierickx CC, Gonzalez S, Lin TY, Campos VB, Gonzalez E, Anderson RR. Laser hair removal affects sebaceous glands and sebum excretion: a pilot study. J Am Acad Dermatol 1999; 41(2 Pt 1):176-180. 
17. Nordstrom KM, Labows JN, McGinley KJ, Leyden JJ. Characterization of wax esters, triglycerides, and free fatty acids of follicular casts. J Invest Dermatol 1986; 86(6):700-705.

18. Murphy GF. Chapter 3. Histology of the skin. In: Elder D, Elenitsas R, Jaworsky C, Jr. BJ, eds. Lever's histopathology of the skin. 8 th edition ed. Philadelphia, PA: Lippincott-Raven Publishers. 1997:1073.

19. Wang L, Jacques SL, Zheng L. MCML--Monte Carlo modeling of light transport in multi-layered tissues. Comput Methods Programs Biomed 1995; 47(2):131146.

20. Jacques S, Alter C, Prahl S. Angular dependence of HeNe laser light scattering by human dermis. . Lasers in the Life Sciences 1987; 1:309-334.

21. Bolin FP, Preuss LE, Taylor RC, Ference RJ. Refractive index of some mammalian tissues using a fiber optic cladding method. Appl Opt 1989; 28(12):2297-2303.

22. Prahl SA, van Gemert MJ, Welch AJ. Determining the optical properties of turbid mediaby using the adding-doubling method. Appl Opt 1993; 32(4):559-568.

23. Williams G. JLab FEL Specifications. Volume 2008. Newport News.

24. Reiken SR, Wolfort SF, Berthiaume F, Compton C, Tompkins RG, Yarmush ML. Control of hypertrophic scar growth using selective photothermolysis. Lasers Surg Med 1997; 21(1):7-12.

25. Thomsen S, Pearce JA, Cheong WF. Changes in birefringence as markers of thermal damage in tissues. IEEE Trans Biomed Eng 1989; 36(12):1174-1179.

26. Neumann RA, Knobler RM, Pieczkowski F, Gebhart W. Enzyme histochemical analysis of cell viability after argon laser-induced coagulation necrosis of the skin. J Am Acad Dermatol 1991; 25(6 Pt 1):991-998.

27. Balasubramaniam $\mathrm{T}$, Bowman $\mathrm{H}$. Thermal conductivity and thermal diffusivity of biomaterials: a simultaneous measurement technique. J Biomech Eng 1977; 99 148-154.

28. Weyer L, Lo S-C. Spectra-Structure Correlations in the Near-infrared. U.K.: John Wiley \& Sons, Ltd. 2002.

29. Alexander VV, Ke K, Xu Z, Islam MN, Freeman MJ, Pitt B, Welsh MJ, Orringer JS. Photothermolysis of sebaceous glands in human skin ex vivo with a $1,708 \mathrm{~nm}$ Raman fiber laser and contact cooling. Lasers Surg Med 2011; 43(6):470-480.

30. Sakamoto FH, Doukas A, Farinelli W, Tannous Z, Shinn M, Benson S, Williams G, Dylla F, Anderson RR. Selective photothermolysis to target sebaceous glands. Lasers in Surgery and Medicine 2008:32-32. 


\section{Legends}

Figure 1. Measured absorption coefficients $\left(\mu_{\mathrm{a}}\right)$ in $\mathrm{cm}^{-1}$ of artificial sebum (AS) and water For AS the $28 \%(\mathrm{w} / \mathrm{w})$ solution and $1 \mathrm{~mm}$ pathlength cuvette used, $\mu_{\mathrm{a}}=23$ O.D. $/$ 0.25 , where O.D. is the measured optical density.

Figure 2. Photothermal excitation of human facial skin, water and artificial sebum at different wavelengths in the infrared spectrum.

Figure 3. Human skin frozen section obtained with NBTC after $1700 \mathrm{~nm}$ FEL exposure at 125 ms pulse duration. Left side was exposed to the laser beam, showing weaker NBTC blue staining consistent with thermal damage after laser irradiation. Sebaceous glands stain strongly with NBTC compared to epidermis; there was no clear evidence of selective sebaceous gland injury by NBTC stain.

Ep: epidermis; PSU: pilosebaceous unit.

Figure 4. H\&E slide of human skin (4x) after FEL exposure at $1700 \mathrm{~nm}, 175 \mathrm{~ms}, 117$ $\mathrm{J} / \mathrm{cm}^{2}$. (A) Laser exposure is on the right side of the slide, showing non-selective thickening and homogenization of collagen bundles consistent with non-selective thermal damage of dermis, and subepidermal blister. (B) Although complete loss of birefringence was not observed, some reduction on the irradiated area after polarized microscopy could correspond to thermal damage of collagen type I.

Figure 5. H\&E slides at 10x. (A) control; (B) $1700 \mathrm{~nm}$ laser exposed sites at $100 \mathrm{~ms}$, (C) $125 \mathrm{~ms}$ and (D) $135 \mathrm{~ms}$. Thicken and hyperchromatic collagen bundles around hair 
follicles (HF) and loss of structure of sebocytes are consistent with selective photothermolysis of sebaceous glands (arrows). There was no evidence of epidermal or superficial dermal injury.

\section{Acknowledgments}

The authors thank Joseph A. Gardecki, Ph.D. and Alexandra Cho, Ph.D. of the Wellman Center for Photomedicine, Massachusetts General Hospital, and Scott Prahl, Ph.D. of the Biomedical Optics Group of Oregon University for stimulating the discussion; Francesca Rius for assistance with statistical analysis; Jinze Qiu, Ph.D. for assistance with the Monte Carlo simulation and Beverly Dammin for general support.

\section{Disclosure of Proprietary Interests}

None of the authors have a conflict of interest regarding this publication. 
Table 1. Estimated exposure parameters for selective photothermolysis of lipids structures.

\begin{tabular}{|c|c|c|}
\hline & Subcutaneous fat & Sebaceous glands \\
\hline Size $(\mathrm{mm})$ & $>5$ (typically) & $0.26 \pm 0.11^{*}$ \\
\hline Thermal relaxation time $(\tau)$ (seconds) & $>20$ (typically) & $\sim 0.06-0.100^{*}$ \\
\hline Depth of location (mm) & $\sim 2.0$ (typically) & $0.5 \pm 0.2$ \\
\hline$\rho c\left(\mathrm{~J} \mathrm{~cm}^{-3} .{ }^{\circ} \mathrm{C}^{-1}\right)$ & $\sim 2.1^{(27)}$ & $\sim 3.57$ \\
\hline Temperature raise $(\Delta \mathrm{T})\left({ }^{\circ} \mathrm{C}\right)$ & 20 & 50 \\
\hline \multicolumn{3}{|l|}{$\sim 1210 \mathrm{~nm}$ band } \\
\hline$\mu_{a}\left(\mathrm{~cm}^{-1}\right)$ & $1.52^{(15)}$ & $2.2^{*}$ \\
\hline Transmittance to target $(T)$ & $\sim 15.6 \%{ }^{\S}$ & $\sim 33.1 \%{ }^{\S}$ \\
\hline Estimated fluence $\left(\mathrm{J} / \mathrm{cm}^{2}\right)$ & $\sim 120^{(15)} \sim 177^{* *}$ & $\sim 245^{* *}$ \\
\hline \multicolumn{3}{|l|}{$\sim 1720 \mathrm{~nm}$ band } \\
\hline$\mu_{a}\left(\mathrm{~cm}^{-1}\right)$ & $10.32^{(15)}$ & $8.1^{*}$ \\
\hline Transmittance to target $(T)$ & $11 \%$ & $32.8 \%{ }^{\S}$ \\
\hline Estimated fluence $\left(\mathrm{J} / \mathrm{cm}^{2}\right)$ & $\sim 37^{* *}$ & $\sim 67^{* *}$ \\
\hline
\end{tabular}

$\mu_{a}:$ absorption coefficient.

* Data from this study.

${ }^{* *}$ Estimated incident fluence $\left(\mathrm{F}_{\mathrm{o}}\right), \mathrm{F}_{\mathrm{o}} \approx \Delta \mathrm{T} . \rho \mathrm{c} / \mu_{\mathrm{a}} . T$; where $\Delta \mathrm{T}$ : temperature raise, $\rho \mathrm{c}$ : heat capacity, $\mu_{\mathrm{a}}$ : coefficient of absorption, $T$ : transmittance through the skin.

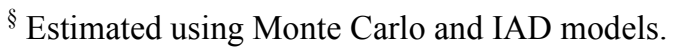


Measured absorption coefficients ( $\mu \mathrm{a}$ ) in $\mathrm{cm}-1$ of artificial sebum (AS) and water For AS the 28\% (w/w) solution and $1 \mathrm{~mm}$ pathlength cuvette used, $\mu \mathrm{a}=23$ O.D./ 0.25, where O.D. is the measured optical density.

$27 \times 21 \mathrm{~mm}(300 \times 300 \mathrm{DPI})$

John Wiley \& Sons, Inc. 
Photothermal excitation of human facial skin, water and artificial sebum at different wavelengths in the infrared spectrum. $122 \times 90 \mathrm{~mm}(300 \times 300$ DPI $)$

John Wiley \& Sons, Inc. 
Human skin frozen section obtained with NBTC after $1700 \mathrm{~nm}$ FEL exposure at 125 ms pulse duration. Left side was exposed to the laser beam, showing weaker NBTC blue staining consistent with thermal damage after laser irradiation. Sebaceous glands stain strongly with NBTC compared to epidermis; there was no clear evidence of selective sebaceous gland injury by NBTC stain.

Ep: epidermis; PSU: pilosebaceous unit.

$101 \times 130 \mathrm{~mm}(300 \times 300$ DPI $)$ 


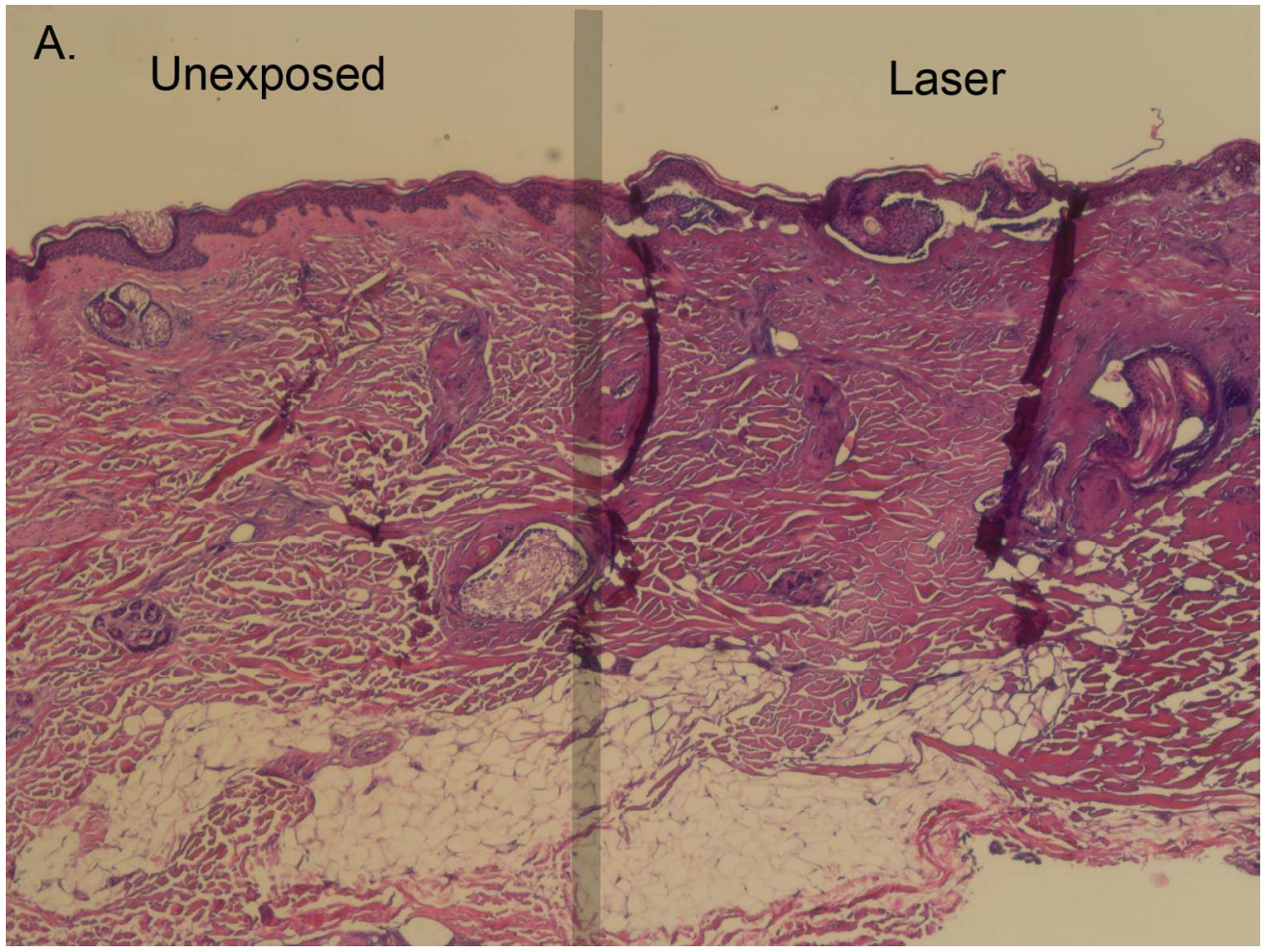

H\&E slide of human skin (4x) after FEL exposure at $1700 \mathrm{~nm}, 175 \mathrm{~ms}, 117 \mathrm{~J} / \mathrm{cm} 2$. (A) Laser exposure is on the right side of the slide, showing non-selective thickening and homogenization of collagen bundles consistent with non-selective thermal damage of dermis, and subepidermal blister. $564 \times 423 \mathrm{~mm}(72 \times 72 \mathrm{DPI})$ 


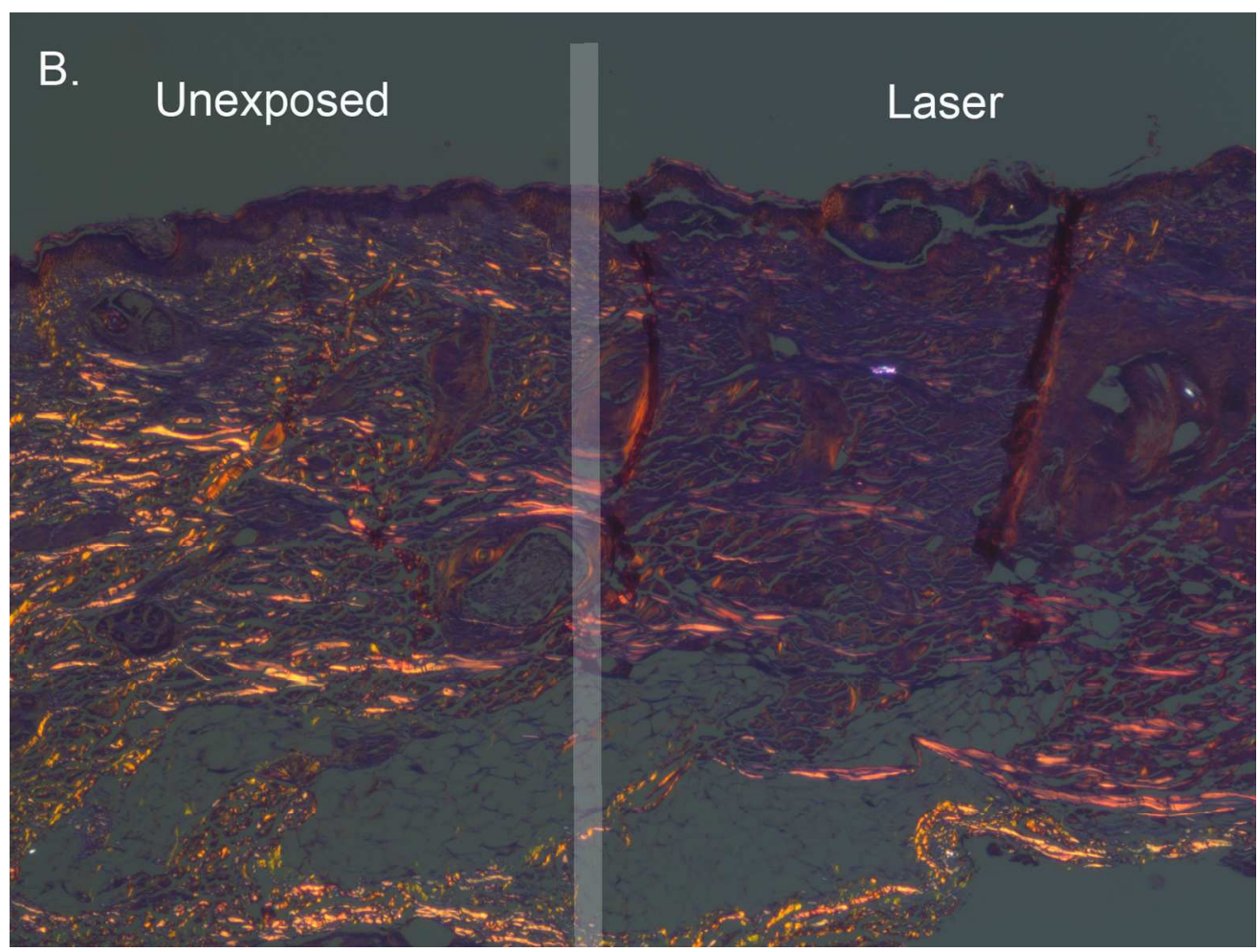

(B) Although complete loss of birefringence was not observed, some reduction on the irradiated area after polarized microscopy could correspond to thermal damage of collagen type I. $564 \times 423 \mathrm{~mm}(72 \times 72 \mathrm{DPI})$ 


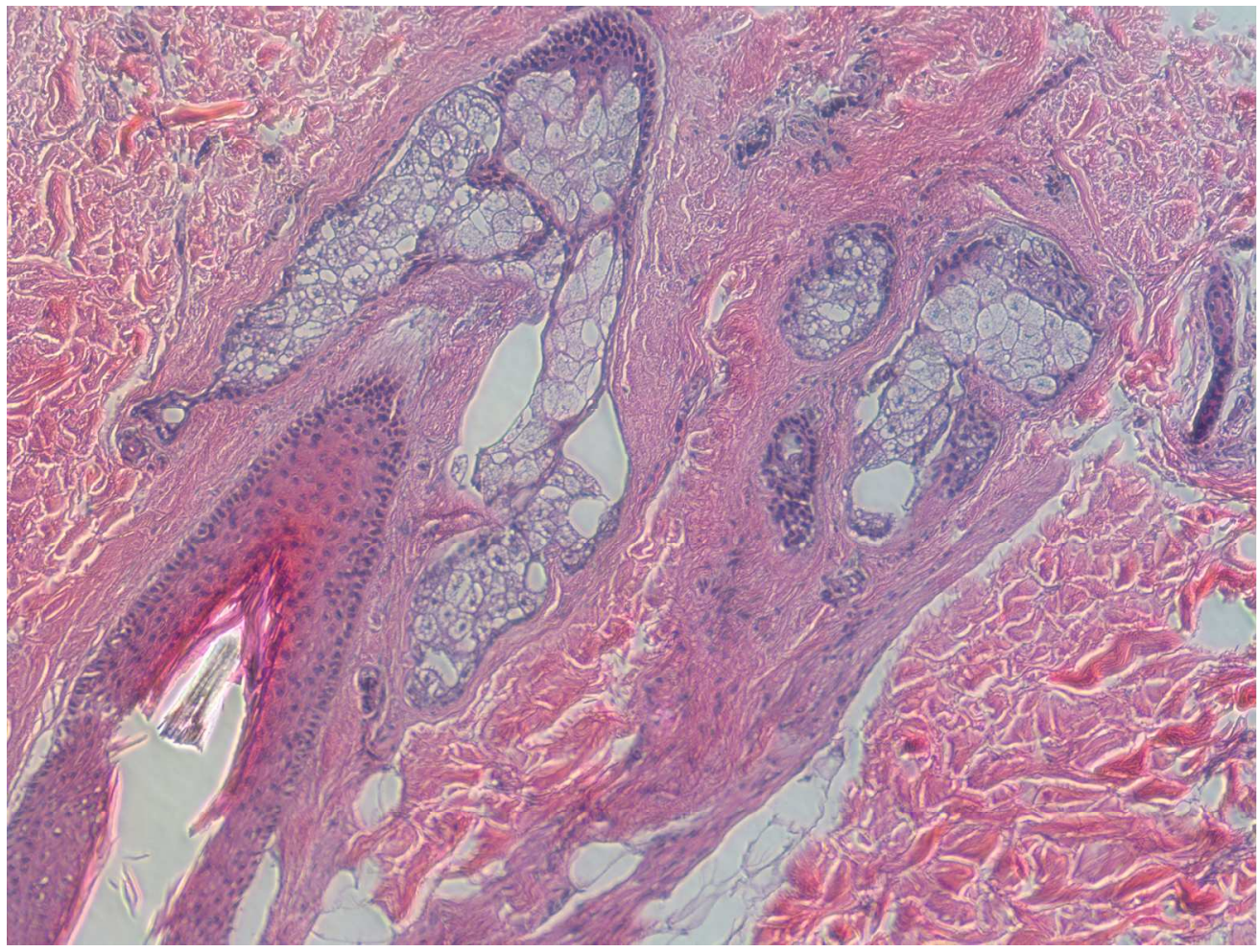

H\&E slides at 10x. (A) control; $564 \times 423 \mathrm{~mm}(72 \times 72$ DPI)

John Wiley \& Sons, Inc. 


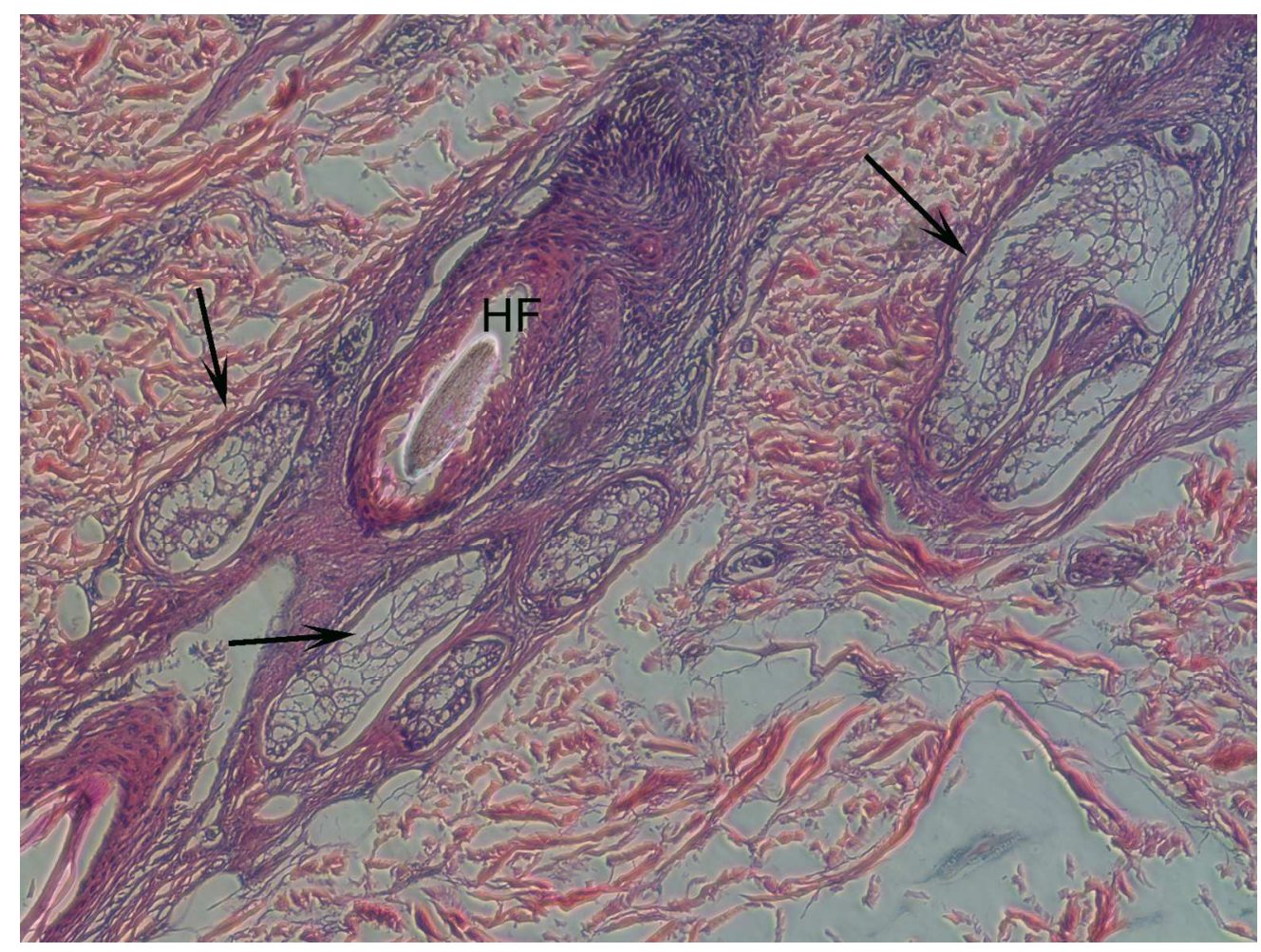

(B) $1700 \mathrm{~nm}$ laser exposed sites at $100 \mathrm{~ms}$, $564 \times 423 \mathrm{~mm}(72 \times 72$ DPI $)$

John Wiley \& Sons, Inc. 


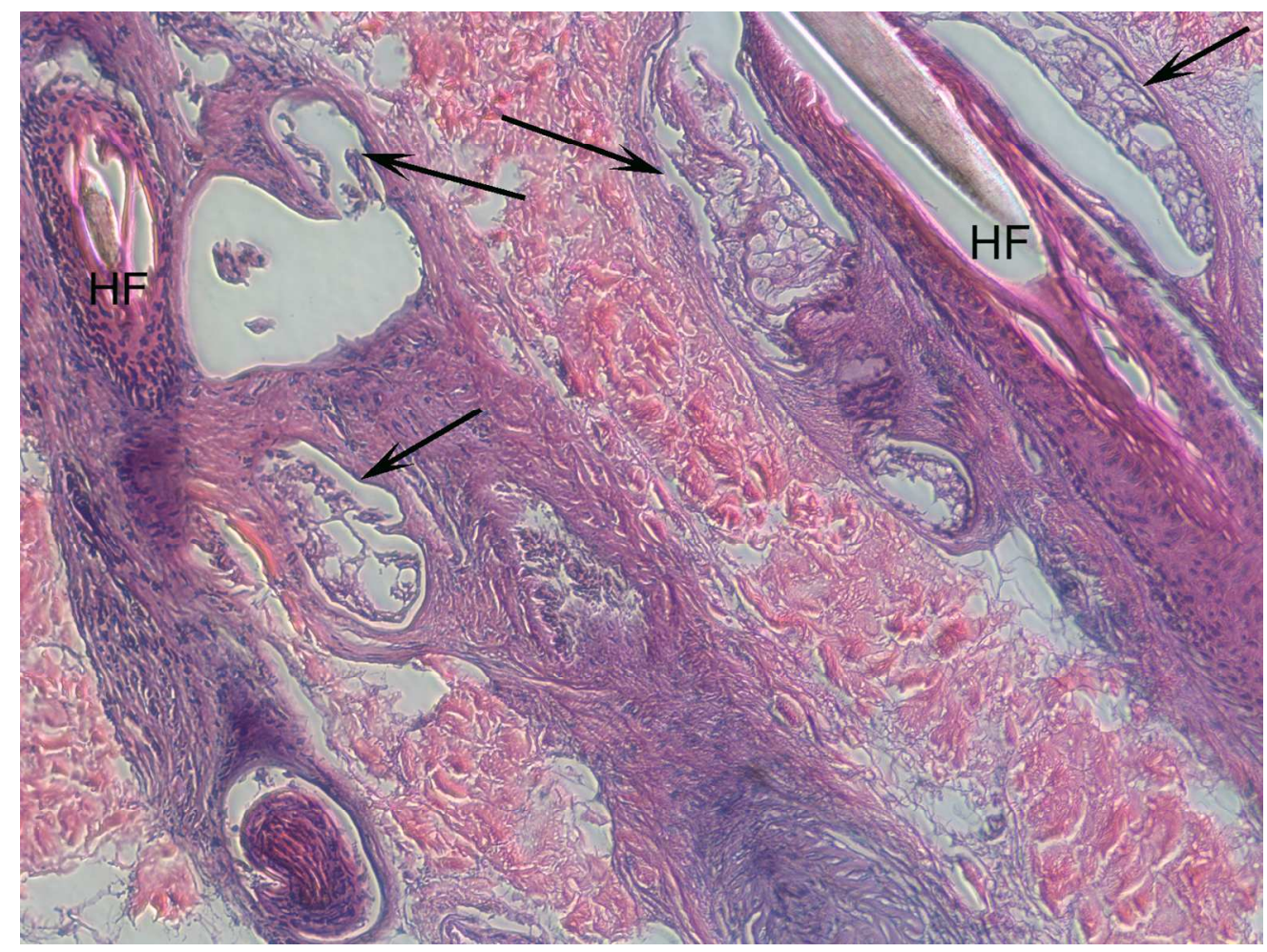

(C) $125 \mathrm{~ms}$

$564 \times 423 \mathrm{~mm}(72 \times 72$ DPI $)$

John Wiley \& Sons, Inc. 


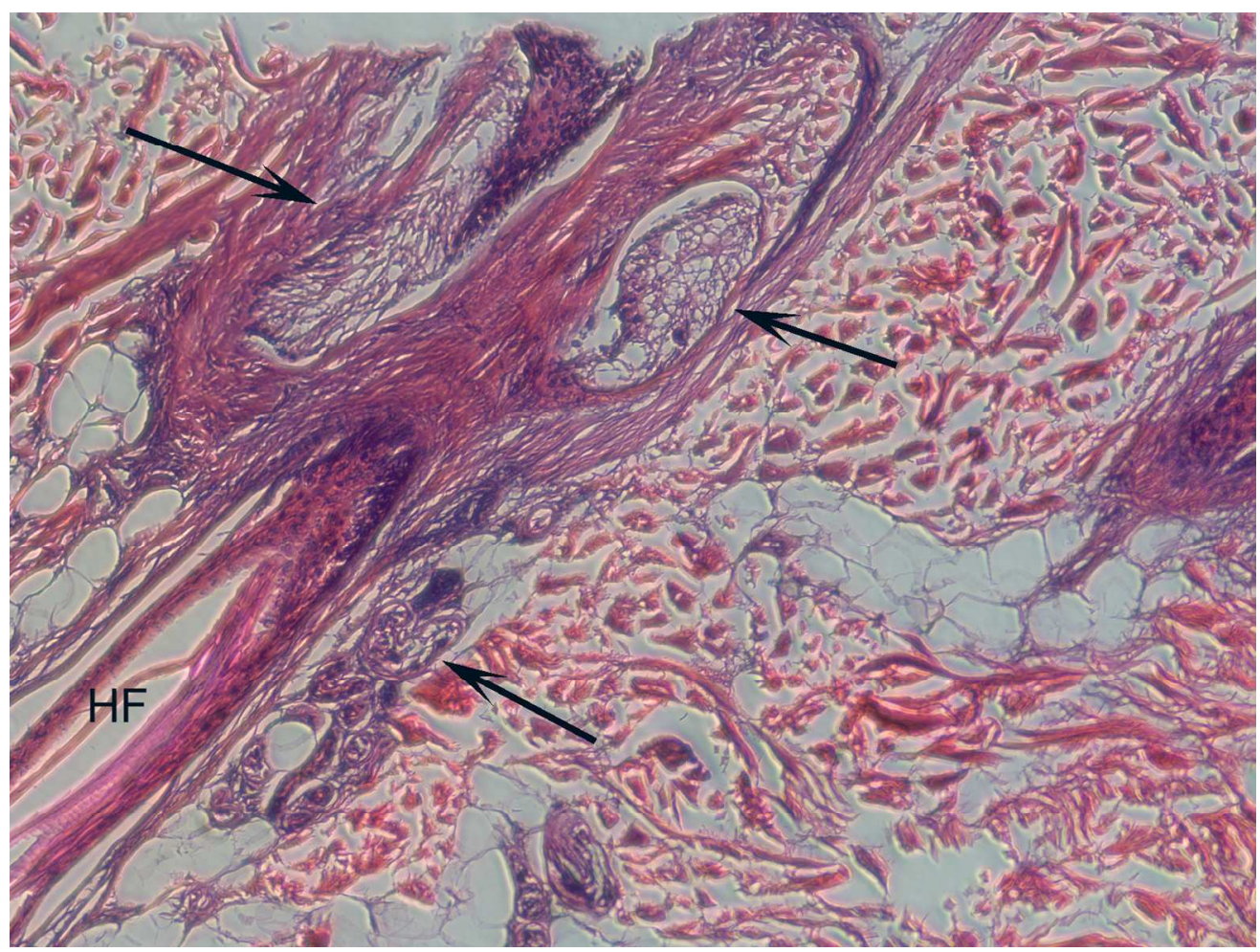

(D) 135 ms. Thicken and hyperchromatic collagen bundles around hair follicles (HF) and loss of structure of sebocytes are consistent with selective photothermolysis of sebaceous glands (arrows). There was not evidence of epidermal or superficial dermal injury.

$564 \times 423 \mathrm{~mm}(72 \times 72 \mathrm{DPI})$ 